\title{
The pharmacology and mechanisms of action of atypical antipsychotic drugs: dopamine and beyond David Sanger*
}

\author{
Address: Sanofi-Aventis Research, Bagneux, France, European Editor, Behavioural Pharmacology
}

* Corresponding author

from International Society on Brain and Behaviour: 2nd International Congress on Brain and Behaviour Thessaloniki, Greece. 17-20 November 2005

Published: 28 February 2006

Annals of General Psychiatry 2006, 5(Suppl I):S34 doi:I0.II86/I744-859X-5-SI-S34

The essential defining feature of atypical antipsychotic drugs is that they are efficacious against the symptoms of schizophrenia but produce fewer motor side effects (EPS) than classical drugs. In addition, there is evidence that atypical agents may be more effective than classical neuroleptics in treatment resistant patients and patients with predominantly negative symptoms. All currently used antipsychotics, both typical and atypical, have affinity for the D2 family of dopamine receptors, at which they act as antagonists. Nevertheless, because some atypical agents, including clozapine, also bind to receptors for other neurotransmitters, particularly serotonin, it has been proposed that such additional mechanisms may be of particular importance in producing an atypical pharmacological profile. However, there is evidence that selective action at dopaminergic mechanisms in certain brain regions can also give rise to antipsychotic efficacy with a very low risk of EPS. Although most atypical drugs have affinities for a variety of neurotransmitter receptors, two drugs, amisulpride and aripiprazole, have been proposed as selective dopaminergic agents. Amisulpride is a selective antagonist at dopamine D2 and D3 receptors whereas aripiprazole may act through partial agonism at D2 receptors. The good efficacy and safety of these drugs raise questions about the roles of neurotransmitters other than dopamine. Nevertheless, a number of ongoing research programmes are currently underway in the search for the next generation of antipsychotics. While many of these programmes target sites other than dopamine receptors (including receptors for glutamate and neuropeptides), it seems likely that indirect modulation of dopaminergic neurotransmission will play a major role in their mechanisms of action. 